\title{
EFEITO DE AGROTÓXICOS SOBRE Calepitrimerus vitis (NALEPA, 1905) (ACARI: ERIOPHYIDAE) NA REGIÃO DA CAMPANHA DO RIO GRANDE DO SUL ${ }^{1}$
}

\author{
PAULO RICARDO EBERT SIQUEIRA ${ }^{2}$, ANDERSON DIONEI GRÜTZMACHER ${ }^{3}$, \\ UEMERSON SILVA DA CUNHA ${ }^{4}$, ROSETE GOTTINARI KOHN ${ }^{5}$, EDVARD THEIL KOHN ${ }^{6}$
}

RESUMO - O Rio Grande do Sul apresenta a maior área de cultivo de videiras no Brasil; neste Estado, a Região da Campanha possui características edafoclimáticas adequadas à produção de cultivares viníferas de origem europeia e apresentou, na primeira década deste milênio, significativa expansão de área. A partir de 2005, o ácaro-da-ferrugem-da-videira, Calepitrimerus vitis, passou a ser encontrado com frequência nos vinhedos da Campanha. Nos países onde esta espécie está estabelecida, reduções significativas na produção de uva são observadas, e a necessidade de controle é constante, não havendo, até o momento, produtos autorizados no Brasil para o controle de C. vitis. O presente trabalho objetivou avaliar diversos agrotóxicos quanto à eficiência de controle de C. vitis em um vinhedo comercial, na região da Campanha do Rio Grande do Sul. O experimento foi conduzido durante os anos de 2008 e 2009, comparando o nível de infestação nas plantas através do emprego de armadilhas adesivas. No ano de 2008, a eficiência de controle durante o outono não diferiu da testemunha. O emprego de enxofre em uma única pulverização no outono, ou em duas pulverizações, sendo uma no outono e outra na primavera, controlou eficientemente $C$. vitis na primavera. Os acaricidas abamectina e espirodiclofeno foram eficientes no controle de $C$. vitis na primavera, tanto com uma única pulverização, como com duas pulverizações, sendo uma no outono e outra na primavera. No outono de 2009, os tratamentos com espirodiclofeno e cihexatina foram eficientes no controle de C. vitis. O ácaro-da-ferrugem-da-videira é controlado eficientemente com pulverizações de cihexatina, enxofre ou espirodiclofeno no outono ou durante a primavera com abamectina, enxofre ou espirodiclofeno.

Termos para indexação: Controle Químico, Acaricidas, Tratamento de Outono, Vitis vinifera, Ácaro-daferrugem-da-videira.

\section{EFFECT OF PESTICIDE ON Calepitrimerus vitis (NALEPA, 1905) (ACARI: ERIOPHYIDAE) IN CAMPANHA REGION FROM THE STATE OF RIO GRANDE DO SUL, BRAZIL}

\begin{abstract}
Rio Grande do Sul has the largest area of vine cultivation on Brazil, in this State, Campanha region has soil and climate suitable characteristics to produce grapes cultivars of European origin and presented in the first decade of this millennium a great expansion in area. Since 2005, the grape rust mite Calepitrimerus vitis is now frequently found in vineyards in Campanha region. In countries where this species is established, significant reductions on grape production are observed and the need for control is constant, so far there are not approved products in Brazil for the $C$. vitis control. This study aimed to evaluate various pesticides on the efficiency of $C$. vitis control in a commercial vineyard in Campanha region from Rio Grande do Sul. The experiment was conducted during the years 2008 and 2009 comparing the level of infestation in plants using sticky traps. In 2008, the level of control during the fall did not differ from the control. The sulfur used in a single spray in autumn or in two sprays, being one in the fall and another in the spring, was efficiently to control C. vitis in the spring. Abamectin and spirodiclofen acaricides were efficient in $C$. vitis controlling in spring, applying only a single spray in the spring or applying two sprays, one in the autumn and another in the spring, in the fall of 2009, the miticides cyhexatin and spirodiclofen treatments were effective in C. vitis controlling. The grape-rust-mite is effectively controlled with cihexatin, sulfur or spirodiclofen pulverizations in the fall or abamectin, sulfur or spirodiclofen sprays during the spring.

Index terms: Chemical control, Acaricides, autumn treatments, Vitis vinifera, Grape-rust- mite.
\end{abstract}

\footnotetext{
${ }^{1}$ (Trabalho 080-11). Recebido em: 21-02-2011. Aceito para publicação em: 04-01-2012.

${ }^{2}$ Eng. Agr. Dr., Prof. do Curso de Agronomia/URCAMP, Bagé-RS. e-mail: siqagro@uol.com.br;

${ }^{3}$ Eng. Agr. Dr., Prof. Depto. Fitossanidade/FAEM/UFPel, Pelotas-RS. e-mail: anderson.grutzmacher@pq.cnpq.br

${ }^{4}$ Eng. Agr. Dr., Prof. Depto. Fitossanidade/FAEM/UFPel, Pelotas-RS. e-mail: uscunha@yahoo.com.br

${ }^{5}$ Eng. Agr. M.Sc., Prof. do Curso de Agronomia/ URCAMP, Bagé-RS. e-mail: rkohn@ibest.com.br

${ }^{6}$ Eng. Agr., Seival State, Candiota-RS, e-mail: viticulturaseival@fortalezadoseival.com.br
} 


\section{INTRODUÇÃO}

Diversos ácaros fitófagos estão associados à videira no Brasil, destacando-se Allonychus braziliensis (Mcgregor), Oligonychus mangiferus Rahman \& Pundjab, Tetranychus cinnabarinus (Boisduval) (Acari: Tetranychidae), Polyphagotarsonemus latus (Banks) (Acari: Tarsonemidae) e Colomerus vitis (Pagenstecher) (Acari: Eriophyidae) (SORIA; DAL CONTE, 2005). Mais recentemente, Panonychus ulmi (Koch) (Acari: Tetranychidae), foi associado a vinhedos da cultivar Merlot no Estado do Rio Grande do Sul (FERLA; BOTTON, 2008).

O ácaro-da-ferrugem-da-videira Calepitrimerus vitis (Nalepa) (Acari: Eriophyidae) está presente em vinhedos de Vitis vinifera (L.) em várias partes do mundo, causando atraso no desenvolvimento e secamento de flores, folhas e ramos (HLUCHÝ; POSPÍŠIL, 1992), promovendo perdas na produção de uvas de até $13 \%$ na Austrália (BERNARD et al., 2003a) a 24\% nos Estados Unidos (WALTON et al., 2007). No Brasil, esta espécie foi relatada inicialmente em 1951, no Rio Grande do Sul, causando danos à cultura e, após um período sem ocorrência, voltou a ser encontrada na safra de 2004/2005, em vinhedos, na região da Campanha, no mesmo Estado (MORAES; FLECHTMANN, 2008).

De acordo com Duso et al. (2010), C. vitis é um caso típico de praga induzida por agrotóxicos, sendo que a redução do número de aplicações de agrotóxicos e a escolha de produtos seletivos aos ácaros predadores são fundamentais para o seu manejo. No Brasil, não existem produtos autorizados para uso no controle de C. vitis (AGROFIT, 2010), sendo importante a avaliação de acaricidas seletivos aos inimigos naturais e com menor impacto negativo ao meio ambiente. Em outros países, o manejo de $C$. vitis é realizado com enxofre antes da abertura das gemas, objetivando controlar as formas deutóginas antes da realização das posturas (BERNARD et al., 2003a) e após a abertura das folhas, visando a controlar as primeiras protóginas, com o uso de acrinatrina, bromopilato, carbaril, dicofol, endossulfano, espirodiclofeno, fempiroximato, fenaziquim e óleo mineral emulsionável (RODRÍGUEZ et al., 2008; DUSO et al., 2010).

$\mathrm{Na}$ Espanha, como alternativa a produtos sintéticos, o emprego de quatro aplicações de óleo de peixe ou óleo de soja, em intervalos de 14 dias, reduziu significativamente a população de $C$. vitis (HÉRNANDEZ RIESGO et al., 2002), enquanto na Austrália o óleo de canola tem sido empregado associado ao enxofre (BERNARD et al., 2003b).

Com base no exposto, objetivou-se avaliar diversos agrotóxicos quanto à eficiência de controle de $C$. vitis em um vinhedo comercial com a cultivar Cabernet Sauvignon, na região da Campanha do Rio Grande do Sul.

\section{MATERIAL E MÉTODOS}

$\mathrm{O}$ experimento foi conduzido durante dois anos agrícolas, em vinhedo comercial da cultivar $\mathrm{Ca}$ bernet Sauvignon, situado no município de Candiota $\left(-31^{\circ} 23^{\prime} 58,5^{\prime}\right.$ ', $-53^{\circ} 45^{\prime} 47,0^{\prime}$ '), na região da Campanha do Rio Grande do Sul. O vinhedo apresenta uma área total de 7,35 hectares e foi implantado em 2002, com espaçamento de 3,00 m entre as fileiras e $1,10 \mathrm{~m}$ entre as plantas, as quais são conduzidas em espaldeira no sistema de cordão esporonado.

Primeiro experimento (2008). Empregouse o delineamento de blocos ao acaso, com parcelas subdivididas e quatro repetições, sendo avaliados os tratamentos de inseticidas/acaricidas nas respectivas dosagens de ingrediente ativo (i.a.) e produto comercial por 100 litros de água: a) abamectina $1,44 \mathrm{~g}$ (Vertimec $18 \mathrm{CE}, 80 \mathrm{~mL}$ ); b) azadiractina 3,6g (Neem Azal-T/S, 300mL); c) enxofre 400g (Kumulus DF, 500g); d) espirodiclofeno 6g (Envidor $240 \mathrm{SC}, 25 \mathrm{~mL}$ ), e e) testemunha sem aplicação, em três épocas de pulverização: outono, primavera e outono+primavera. Cada bloco foi constituído por 48 fileiras, cada uma com 84 plantas, das quais a $9^{\mathrm{a}}, 17^{\mathrm{a}}$, $25^{\mathrm{a}}, 33^{\mathrm{a}}$ e $40^{\mathrm{a}}$ fileiras receberam um dos tratamentos, ficando as demais como proteção antideriva. Nas fileiras que receberam tratamentos, foram demarcadas as unidades experimentais, constituídas por 12 plantas entre dois vãos consecutivos, cada qual com seis plantas. No interior das fileiras, as parcelas foram alocadas entre o $3^{\circ}$ e $4^{\circ}, 7^{\circ}$ e $8^{\circ}$ e $11^{\circ}$ e $12^{\circ}$ vãos, nas quais foram realizadas, em uma destas, a aplicação no outono, em outra, aplicações no outono e na primavera e, na terceira, somente na primavera. A aplicação de outono foi realizada em 30 de abril de 2008, no período pós-colheita e de senescência das folhas; já a aplicação de primavera foi realizada em 03 de outubro de 2008, no estádio de 3 a 4 folhas distendidas. As aplicações foram feitas por meio de um atomizador tratorizado marca Jacto, modelo Airbus 400, equipado com duas pistolas providas de pontas modelo $\mathrm{Y} 8$, operando à pressão de $90 \mathrm{lbf} \mathrm{pol}^{-2}$, sendo a aplicação realizada em ambos os lados das fileiras, até o ponto de escorrimento, com volume de calda de $570 \mathrm{~L} \mathrm{ha}^{-1}$.

A avaliação foi realizada através da amostragem de armadilhas adesivas constituídas por segmentos de fita adesiva dupla face, com $11 \mathrm{~mm}$ de largura, da marca Adelbras ${ }^{\circledR}$, instaladas abaixo da 
segunda gema, nos primeiros ramos de produção, de modo a circundar estes ramos, as quais permaneceram nas plantas por 72 horas. Em cada repetição, foram amostradas 10 plantas, desprezando-se a primeira e a última, consideradas como bordaduras, e de cada planta foi coletada uma armadilha. Foi realizada uma avaliação prévia à aplicação dos tratamentos, através do recolhimento de armadilhas, as quais haviam sido instaladas com antecedência de 72 horas, e outra, aos 21 dias após o tratamento (DAT), nas parcelas que receberam o tratamento de outono.

Nas avaliações da primavera de 2008, as armadilhas adesivas foram recolhidas antes da aplicação (0 DAT), após um período de 72 horas no vinhedo; para as demais datas (7 e 21 DAT), considerou-se o dia da instalação das armadilhas.

Segundo experimento (2009). Empregou-se o delineamento de blocos completos inteiramente casualizados e quatro repetições, sendo seis os tratamentos de inseticida/acaricida, nas respectivas dosagens de ingrediente ativo e produto comercial por 100 litros de água: a) abamectina 1,44g (Vertimec $18 \mathrm{CE}, 80 \mathrm{~mL})$; b) azadiractina 3,6g (Neem Azal-T/S, $300 \mathrm{~mL}$ ); c) cihexatina $30 \mathrm{~g}$ (Sipcatin $500 \mathrm{SC}, 60 \mathrm{~mL}$ ); d) enxofre $400 \mathrm{~g}$ (Kumulus DF, 500g); e) espirodiclofeno 7,2g (Envidor $240 \mathrm{SC}, 30 \mathrm{~mL}$ ), e f) testemunha sem aplicação. Cada bloco foi constituído por uma fileira de plantas, mantendo-se, entre um bloco e outro, oito fileiras como bordaduras. Cada unidade experimental correspondeu às 12 plantas compreendidas entre dois vãos consecutivos, sendo os tratamentos distribuídos ao acaso, em cada bloco, havendo a manutenção de 12 plantas sem tratamentos, entre uma unidade experimental e outra, para servirem de bordadura, sendo avaliadas as 10 plantas centrais, pela coleta de uma armadilha adesiva, conforme o ano anterior. A aplicação foi realizada em 22 de abril de 2009, com pulverizador costal, à pressão de 15 lbf pol$^{-2}$, e ponta do tipo cone vazio, modelo MAG 6 , com volume de calda de $790 \mathrm{~L} \mathrm{ha}^{-1}$. As avaliações foram realizadas aos 0; 4; 10 e 16 DAT.

Análise estatística. As observações foram transformadas segundo $\sqrt{\mathrm{x}+1,0}$, sendo os resultados analisados pelo teste $\mathrm{F}$, com a comparação das médias pelo teste de Tukey, ao nível de 5\% de probabilidade, utilizando-se do programa Statistica for Windows v.7. Para determinar a eficiência de controle dos tratamentos, foi empregada a fórmula de Henderson e Tilton (1955).

\section{RESULTADOS E DISCUSSÃO}

Primeiro experimento (2008). Nas avaliações realizadas no outono, tanto no dia da pulveri- zação (0 DAT) como aos 21 DAT, não foram encontradas diferenças significativas entre os tratamentos para número de $C$. vitis capturados nas armadilhas adesivas (Tabela 1). A reduzida eficiência dos produtos pode ser atribuída à ocorrência de chuva dois dias após a pulverização.

$\mathrm{Na}$ avaliação realizada em três de outubro de 2008 (0 DAT), o número de capturas de $C$. vitis nas armadilhas adesivas não diferiu significativamente entre os tratamentos da época "outono", pulverizados há 155 dias, bem como entre os tratamentos da época "primavera", nessa oportunidade, ainda sem pulverização. Nas plantas da época "outono+primavera", nessa ocasião somente com a pulverização de outono, os acaricidas enxofre e espirodiclofeno reduziram a população de $C$. vitis e diferiram significativamente do tratamento com abamectina (Tabela 2).

As aplicações de enxofre e de espirodiclofeno no outono contribuíram para reduzir a população das formas hibernantes de $C$. vitis, as quais naturalmente sofrem uma elevada taxa de mortalidade durante o inverno, de modo que, de cada 30 indivíduos que se dirigem para os sítios de hibernação, somente um sobrevive até a primavera (BERNARD et al., 2003a). $\mathrm{O}$ efeito destes tratamentos, associado à mortalidade natural elucida a ausência $C$. vitis durante a primeira avaliação, na primavera, nas plantas tratadas com espirodiclofeno no outono e outono+primavera e de enxofre nas plantas da época outono+primavera (Tabela 2). Nesta oportunidade, os tratamentos com enxofre e espirodiclofeno, pulverizados no outono e no outono+primavera, reduziram significativamente a infestação de $C$. vitis em relação às plantas da época da primavera, ainda não pulverizadas até esse momento; enquanto nos demais tratamentos, não foram observadas diferenças significativas entre as épocas de pulverização (Tabela 3 ).

Aos 7 DAT de primavera, foi observada uma redução natural nas capturas de $C$. vitis, não ocorrendo diferenças significativas entre os tratamentos (Tabela 2), assim como entre as épocas de pulverização (Tabela 3). Nesta ocasião, o tratamento com enxofre pulverizado no outono e o tratamento com espirodiclofeno pulverizado no outono+primavera apresentaram controle de 100\% (Tabela 2).

Na avaliação de 24 de outubro, aos 21 DAT de primavera, não foi verificada diferença significativa entre os tratamentos pulverizados apenas no outono, sendo observada uma eficiência de $80,9 \%$ no tratamento com azadiractina e de $100 \%$ no tratamento com enxofre. Nas parcelas com aplicação somente na primavera, azadiractina apresentou infestação significativamente superior aos tratamentos com abamectina e espirodiclofeno, os quais 
apresentaram $100 \%$ de eficiência. As capturas nas armadilhas adesivas, nas plantas pulverizadas no outono+primavera, não apresentaram diferenças significativas entre os tratamentos, sendo que abamectina, enxofre e espirodiclofeno apresentaram $100 \%$ de controle (Tabela 2).

Aos 21 DAT, os tratamentos com azadiractina e espirodiclofeno apresentaram infestação de $C$. vitis significativamente menor nas parcelas pulverizadas no outono e no outono+primavera, em relação às plantas pulverizadas exclusivamente na primavera; nos demais tratamentos, a época de aplicação não apresentou diferenças significativas (Tabela 3).

A pulverização de enxofre no outono reduziu acentuadamente a população de $C$. vitis no início da primavera, e esta redução verificou-se durante as avaliações de primavera, com maior evidência aos 21 DAT, quando as plantas tratadas no outono, independentemente do tratamento de primavera, apresentaram $100 \%$ de controle, enquanto naquelas tratadas apenas na primavera, o percentual de controle foi de $70,6 \%$ (Tabela 2). Este fato encontra similaridade com tratamentos de outono para Eriophyes pyri (Pagenstecher) (Acari: Eriophyidae), em pomares de pera na Suíça, após a colheita das frutas, nos quais apenas uma aplicação de enxofre reduziu em $95 \%$ a população de $E$. pyri no outono e na primavera seguinte, diminuindo os danos ao cultivo (DANIEL et al., 2007).

A ação do enxofre sobre as formas móveis de eriofídeos dá-se pela liberação de compostos gasosos asfixiantes, com curto período de ação e sem limitações toxicológicas quanto ao número de tratamentos (CARMONA; DIAS, 1996). No Brasil, Soria e Dal Conte (2005) indicam o enxofre para o controle de Col. vitis durante a primavera e o verão. Nos Estados Unidos, enxofre e espirodiclofeno são empregados para o controle de Phyllocoptruta oleivora (Ashmead) e Aculops pelekassi (Keifer) (Acari: Eriophyidae) em citros no outono, durante o repouso vegetativo, embora as infestações nesta estação sejam inferiores às infestações de verão (ROGERS et al., 2010).

O espirodiclofeno atua no desenvolvimento dos ácaros inibindo a síntese de lipídios com intensa ação ovicida e elevada toxicidade aguda às formas imaturas, e causa nas fêmeas adultas bloqueio da oviposição, resultando na morte até cinco dias devido ao acúmulo de ovos no corpo, como verificado em Tetranychus urticae Koch (Tetranychidae) (WACHENDORFF et al., 2002), além da redução na fecundidade e fertilidade (VAN POTTELBERGE et al., 2009). No Brasil, Reis et al. (2005) verificaram em Brevipalpus phoenicis (Geijskes) (Acari: Tenuipalpidae) eficiência de $100 \%$ em ovos no início do desenvolvimento embrionário e de $98 \%$ ao final deste período. Estas interferências na biologia dos ácaros contribuem para explicar a ausência de $C$. vitis, ao 0 DAT, nas plantas pulverizadas com espirodiclofeno no outono e a eficiência de $100 \%$ aos 7 DAT, nas plantas que receberam aplicações no outono+primavera, e aos 21 DAT, nestas plantas e também naquelas tratadas apenas na primavera (Tabela 2). O inseticida-acaricida abamectina apresentou ação lenta e crescente, sendo que aos 21 DAT demonstrou eficiência de 100\% nas parcelas que receberam tratamentos na primavera $\mathrm{e}$ em ambas as estações (Tabela 2).

$\mathrm{Na}$ primavera, a azadiractina apresentou, aos 21 DAT, a mais elevada infestação entre as parcelas tratadas apenas na primavera, enquanto naquelas em que houve tratamento no outono e no outono+primavera, a infestação não diferiu dos demais tratamentos (Tabela 2). Esta observação evidencia que azadiractina possui ação lenta para a supressão de $C$. vitis, que pode estar associada à ação ovicida, semelhante ao verificado em T. urticae, em que a exposição das posturas ao óleo de nim reduziu a viabilidade dos ovos de $97,5 \%$ para menos de $19 \%$ (BRITO et al., 2006). O óleo de nim é utilizado por produtores de uva orgânica dos Estados Unidos, visando ao controle de Tetranychidae, com exceção no período de maturação das uvas, quando o produto pode afetar negativamente a qualidade dos frutos (DUFOR, 2006).

Segundo experimento (2009). Foi verificado que, aos 4 DAT, os acaricidas cihexatina e espirodiclofeno apresentaram $100 \%$ de eficiência e diferiram significativamente da testemunha e dos demais tratamentos acaricidas, enquanto o tratamento com abamectina foi superior à testemunha e aos tratamentos com azadiractina e enxofre (Tabela 4). Aos 10 DAT, não foram verificadas diferenças significativas no nível de infestação entre os tratamentos (Tabela 4).

Aos 16 DAT, o espirodiclofeno apresentou $66 \%$ de controle e diferiu significativamente do tratamento com azadiractina, sem diferir dos demais tratamentos (Tabela 4). O prolongado período de ação biológica de espirodiclofeno também foi verificado por Vieira et al. (2006), no controle de Calacarus heveae Feres (Acari: Eriophyidae) em seringueira, e por Raudonis (2006), no controle de T. urticae e Tarsonemus pallidus (Banks) (Acari: Tarsonemidae), em morangueiro.

A eficiência observada no acaricida cihexatina encontra respaldo em Carmona e Dias (1996), segundo os quais este produto apresenta elevado controle de Eriophyidae, cujo período de ação pode atingir 
30 dias. Na Argentina, cihexatina causou elevada mortalidade de Neoseiulus californicus (McGregor) (Acari: Phytoseiidae) em macieira, 24 horas após a aplicação; entretanto, com rápida taxa de decréscimo da toxicidade, sendo considerado um produto levemente persistente (RUIZ; MORAES, 2008). Todavia seu uso na Europa deverá ser abandonado por riscos ambientais apontados pela European Pesticide Review Program (VAN LEEUWEN et al., 2010).

Avaliando-se os dois anos de experimentação, verificou-se que, nas aplicações de outono, o espirodiclofeno apresentou os maiores percentuais de controle de $C$. vitis, sendo este resultado importante devido ao desempenho deste acaricida ser melhor em temperatura alta $\left(30^{\circ} \mathrm{C}\right)$, em relação a temperaturas amenas $\left(20^{\circ} \mathrm{C}\right)$ (WACHENDORFF et al., 2002). A eficiência de 100\% verificada no ano de 2008 para espirodiclofeno aos 21 DAT, nas plantas tratadas na primavera, e aos 7 e 21 DAT (Tabela 2), nas plantas tratadas no outono+primavera, utilizando a dosagem de 6 g i.a. $100 \mathrm{~L}^{-1}$, e aos 4 e 10 DAT no outono de 2009, empregando $7,2 \mathrm{~g}$ i.a. $100 \mathrm{~L}^{-1}$, é representativa se comparada àquela obtida por De Lillo et al. (2004), com eficiência de controle de $C$. vitis, entre 58,9 e $88,4 \%$, ao empregarem $30 \mathrm{~g}$ i.a. e entre 81,2 e $92,3 \%$ com a dosagem de $40 \mathrm{~g}$ i.a..

A cihexatina apresentou eficiência similar ao espirodiclofeno aos 4 DAT e aos 10 DAT, com uma redução acentuada de eficiência aos 16 DAT (Tabela 4). Os tratamentos com enxofre e espirodiclofeno pulverizados no outono reduziram significativamente a população de $C$. vitis no início da primavera de 2008, em relação às parcelas pulverizadas com estes produtos apenas na primavera (Tabela 3). Para as aplicações realizadas exclusivamente na primavera, os maiores percentuais de controle ocorreram nos tratamentos com abamectina e espirodiclofeno aos 21 DAT.
A abamectina apresentou baixa eficiência nas aplicações de outono (Tabelas 1 e 4); entretanto, quando este tratamento foi realizado na primavera, foi obtido controle de $100 \%$ aos 21 DAT (Tabela 2). Estes resultados contrastantes podem estar associados à baixa suscetibilidade dos eriofídeos causadores de bronzeamentos à abamectina (VAN LEEUWEN et al., 2010), resultando em desempenhos distintos, conforme as condições do ambiente por ocasião do tratamento.

Considerando a necessidade de estabelecerse um plano de manejo para $C$. vitis, baseado em produtos registrados no Brasil para uso na cultura da videira, o emprego de enxofre no outono ou na primavera e de abamectina na primavera, além de reduzir os problemas decorrentes do ataque do ácaro, poderá minimizar o impacto negativo ao meio ambiente, comparativamente ao controle por meio de pulverizações sucessivas de acaricidas, bem como evitar possíveis problemas de resíduos de agrotóxicos em uvas. Além do mais, as informações obtidas neste trabalho poderão embasar análises mais detalhadas visando ao registro de outros acaricidas na cultura da videira, como o espirodiclofeno, com recomendação de uso, dentre outras culturas, na produção integrada de maçã, na cafeicultura e citricultura (AGROFIT, 2010) e considerado seletivo a inimigos naturais, como os ácaros predadores Euseius citrifolius Denmark \& Muma (Acari: Phytoseiidae) e a Euseius alatus DeLeon (Acari: Phytoseiidae) (REIS et al., 2005), ao parasitoide de ovos de Trichogramma pretiosum Riley (Hymenoptera: Trichogrammatidae) (MANZONI et al., 2006) e aos predadores Chrysoperla externa (Hagen) (Neuroptera: Chrysopidae) e Ceraeochrysa cubana (Hagen) (Neuroptera: Chrysopidae) (GODOY et al., 2010), além de ser largamente empregado em outros países.

TABELA 1 - Número de Calepitrimerus vitis em 10 armadilhas e percentual de controle (EC) de diferentes agrotóxicos aos 0 e 21 dias após tratamento (DAT), no outono de 2008. Candiota-RS.

\begin{tabular}{lcccc}
\hline Tratamento & DC $^{\mathbf{1}}$ & 0 DAT & 21 DAT & EC $^{\mathbf{2}}$ \\
\hline Testemunha & - & $2,8 \pm 0,63 \mathrm{a}$ & $2,5 \pm 0,50 \mathrm{a}$ & - \\
Abamectina & 80 & $3,2 \pm 1,32 \mathrm{a}$ & $4,0 \pm 2,94 \mathrm{a}$ & 0,0 \\
Azadiractina & 300 & $3,0 \pm 0,41 \mathrm{a}$ & $4,5 \pm 0,64 \mathrm{a}$ & 0,0 \\
Enxofre & 500 & $2,5 \pm 0,86 \mathrm{a}$ & $2,0 \pm 0,41 \mathrm{a}$ & 10,4 \\
Espirodiclofeno & 25 & $3,2 \pm 0,95 \mathrm{a}$ & $2,0 \pm 0,92 \mathrm{a}$ & 30,0 \\
\hline
\end{tabular}

${ }^{1}$ Dosagem do produto comercial em g ou mL por 100 litros de água. Médias ( \pm EP) seguidas por letras distintas, na coluna, diferem entre si, pelo teste de Tukey $(\mathrm{P}<0,05)$.

Dados originais, para análise estatística, foram transformados em $\sqrt{\mathrm{x}+1,0}$.

${ }^{2}$ Percentual de eficiência dos agrotóxicos segundo a fórmula de Henderson e Tilton (1955). 
TABELA 2 - Número de Calepitrimerus vitis em 10 armadilhas e percentual de controle (EC) de diferentes agrotóxicos aos 0; 7 e 21 dias após tratamento (DAT), na primavera de 2008. Candiota-RS.

\begin{tabular}{|c|c|c|c|c|c|c|c|}
\hline Tratamento & DC $^{1}$ & Época de aplicação & 0 DAT & 7 DAT & $\mathbf{E C}^{2}$ & 21 DAT & $\mathbf{E C}^{2}$ \\
\hline Testemunha & - & Outono* & $4,5 \pm 1,05 \mathrm{a}$ & $1,3 \pm 0,10 \mathrm{a}$ & - & $4,3 \pm 1,14 \mathrm{a}$ & - \\
\hline Abamectina & 80 & & $2,0 \pm 0,40 \mathrm{a}$ & $1,0 \pm 0,29 \mathrm{a}$ & 0,0 & $1,5 \pm 0,48 \mathrm{a}$ & 21,5 \\
\hline Azadiractina & 300 & & $5,5 \pm 0,63 \mathrm{a}$ & $2,0 \pm 0,40 \mathrm{a}$ & 0,0 & $1,0 \pm 0,29 \mathrm{a}$ & 80,9 \\
\hline Enxofre & 500 & & $0,5 \pm 0,25 \mathrm{a}$ & $0,0 \pm 0,00 \mathrm{a}$ & 100,0 & $0,0 \pm 0,0 \mathrm{a}$ & 100,0 \\
\hline Espirodiclofeno & 25 & & $0,0 \pm 0,00 \mathrm{a}$ & $0,5 \pm 0,25 \mathrm{a}$ & 0,0 & $1,0 \pm 0,58 \mathrm{a}$ & 0,0 \\
\hline Testemunha & - & Primavera & $4,5 \pm 1,05 \mathrm{a}$ & $1,3 \pm 0,10 \mathrm{a}$ & - & $4,3 \pm 1,14 \mathrm{ab}$ & - \\
\hline Abamectina & 80 & & $2,5 \pm 0,25 \mathrm{a}$ & $2,0 \pm 0,70 \mathrm{a}$ & 0,0 & $0,0 \pm 0,0 \mathrm{~b}$ & 100,0 \\
\hline Azadiractina & 300 & & $6,5 \pm 0,25 \mathrm{a}$ & $4,5 \pm 1,90 \mathrm{a}$ & 0,0 & $18,0 \pm 3,74 \mathrm{a}$ & 0,0 \\
\hline Enxofre & 500 & & $16,0 \pm 1,08 \mathrm{a}$ & $2,0 \pm 0,00 \mathrm{a}$ & 56,7 & $4,5 \pm 1,50 \mathrm{ab}$ & 70,6 \\
\hline Espirodiclofeno & 25 & & $2,0 \pm 0,41 \mathrm{a}$ & $1,0 \pm 0,58 \mathrm{a}$ & 0,0 & $0,0 \pm 0,0 \mathrm{~b}$ & 100,0 \\
\hline Testemunha & - & Outono $*+$ Primavera & $4,5 \pm 1,05 \mathrm{ab}$ & $1,3 \pm 0,10 \mathrm{a}$ & - & $4,3 \pm 1,14 \mathrm{a}$ & - \\
\hline Abamectina & 80 & & $6,0 \pm 1,00 \mathrm{a}$ & $1,0 \pm 0,29 \mathrm{a}$ & 42,3 & $0,0 \pm 0,0 \mathrm{a}$ & 100,0 \\
\hline Azadiractina & 300 & & $0,5 \pm 0,25 \mathrm{ab}$ & $2,0 \pm 0,40 \mathrm{a}$ & 0,0 & $3,5 \pm 1,5 \mathrm{a}$ & 0,0 \\
\hline Enxofre & 500 & & $0,0 \pm 0,00 \mathrm{~b}$ & $2,0 \pm 0,70 \mathrm{a}$ & 0,0 & $0,0 \pm 0,0 \mathrm{a}$ & 100,0 \\
\hline Espirodiclofeno & 25 & & $0,0 \pm 0,00 \mathrm{~b}$ & $0,0 \pm 0,00 \mathrm{a}$ & 100,0 & $0,0 \pm 0,0 \mathrm{a}$ & 100,0 \\
\hline
\end{tabular}

${ }^{1}$ Dosagem do produto comercial em g ou $\mathrm{mL}$ por 100 litros de água.

Médias ( \pm EP) seguidas por letras distintas, na coluna (para cada época de aplicação), diferem entre si, pelo teste de Tukey $(\mathrm{P}<0,05)$.

Dados originais, para análise estatística, foram transformados em $\sqrt{\mathrm{x}+1,0}$.

${ }^{2}$ Percentual de eficiência dos agrotóxicos segundo a fórmula de Henderson e Tilton (1955).

* Aplicação em 30 de abril de 2008.

TABELA 3 - Número de Calepitrimerus vitis em 10 armadilhas, em videiras tratadas com diferentes agrotóxicos, em distintas épocas aos 0; 7 e 21 dias após tratamento (DAT), na primavera de 2008. Candiota-RS.

\begin{tabular}{lclccc}
\hline Tratamento & DC $^{\mathbf{1}}$ & Época de aplicação & 0 DAT & 7 DAT & 21 DAT \\
\hline Abamectina & 80 & Outono & $2,0 \pm 0,40 \mathrm{a}$ & $1,0 \pm 0,29 \mathrm{a}$ & $1,5 \pm 0,48 \mathrm{a}$ \\
& & Primavera & $2,5 \pm 0,25 \mathrm{a}$ & $2,0 \pm 0,70 \mathrm{a}$ & $0,0 \pm 0,0 \mathrm{a}$ \\
& Outono + Primavera & $6,0 \pm 1,00 \mathrm{a}$ & $1,0 \pm 0,29 \mathrm{a}$ & $0,0 \pm 0,0 \mathrm{a}$ \\
\hline Azadiractina & 300 & Outono & $5,5 \pm 0,63 \mathrm{a}$ & $2,0 \pm 0,40 \mathrm{a}$ & $1,0 \pm 0,29 \mathrm{~b}$ \\
& & Primavera & $6,5 \pm 0,25 \mathrm{a}$ & $4,5 \pm 1,90 \mathrm{a}$ & $18,0 \pm 3,74 \mathrm{a}$ \\
& & Outono + Primavera & $0,5 \pm 0,25 \mathrm{a}$ & $2,0 \pm 0,40 \mathrm{a}$ & $3,5 \pm 1,5 \mathrm{~b}$ \\
\hline Enxofre & 500 & Outono & $0,5 \pm 0,25 \mathrm{~b}$ & $0,0 \pm 0,00 \mathrm{a}$ & $0,0 \pm 0,0 \mathrm{~b}$ \\
& & Primavera & $16,0 \pm 1,08 \mathrm{a}$ & $2,0 \pm 0,00 \mathrm{a}$ & $4,5 \pm 1,50 \mathrm{a}$ \\
& & Outono + Primavera & $0,0 \pm 0,00 \mathrm{~b}$ & $2,0 \pm 0,70 \mathrm{a}$ & $0,0 \pm 0,0 \mathrm{~b}$ \\
\hline Espirodiclofeno & 25 & Outono & $0,0 \pm 0,00 \mathrm{~b}$ & $0,5 \pm 0,25 \mathrm{a}$ & $1,0 \pm 0,58 \mathrm{a}$ \\
& & Primavera & $2,0 \pm 0,41 \mathrm{a}$ & $1,0 \pm 0,58 \mathrm{a}$ & $0,0 \pm 0,0 \mathrm{a}$ \\
& Outono + Primavera & $0,0 \pm 0,00 \mathrm{~b}$ & $0,0 \pm 0,00 \mathrm{a}$ & $0,0 \pm 0,0 \mathrm{a}$ \\
\hline
\end{tabular}

${ }^{1}$ Dosagem do produto comercial em g ou $\mathrm{mL}$ por 100 litros de água.

Médias ( \pm EP) seguidas por letras distintas, na coluna (para cada tratamento), diferem entre si, pelo teste de Tukey $(\mathrm{P}<0,05)$. Dados originais, para análise estatística, foram transformados em $\sqrt{\mathrm{x}+1,0}$. 
TABELA 4 - Número de Calepitrimerus vitis em 10 armadilhas e percentual de controle (EC) de diferentes agrotóxicos aos $0 ; 4 ; 10$ e 16 dias após o tratamento (DAT). Candiota-RS.

\begin{tabular}{lcccccccc}
\hline Tratamento & DC $^{\mathbf{1}}$ & 0 DAT & 4 DAT & EC $^{2}$ & 10 DAT & EC $^{2}$ & 16 DAT & EC $^{\mathbf{2}}$ \\
\hline Testemunha & & $2,0 \pm 0,40 \mathrm{a}$ & $6,2 \pm 1,49 \mathrm{a}$ & - & $3,5 \pm 1,19 \mathrm{a}$ & - & $1,2 \pm 0,25 \mathrm{ab}$ & - \\
Abamectina & 80 & $0,8 \pm 0,48 \mathrm{a}$ & $2,2 \pm 0,48 \mathrm{~b}$ & 11,1 & $2.5 \pm 0,96 \mathrm{a}$ & 0,0 & $0,5 \pm 0,29 \mathrm{ab}$ & 0,0 \\
Azadiractina & 300 & $1,5 \pm 0,64 \mathrm{a}$ & $7,5 \pm 0,96 \mathrm{a}$ & 0,0 & $3,5 \pm 1,44 \mathrm{a}$ & 0,0 & $1,5 \pm 0,29 \mathrm{a}$ & 0,0 \\
Cihexatina & 60 & $2,0 \pm 0,91 \mathrm{a}$ & $0,0 \pm 0,00 \mathrm{c}$ & 100,0 & $0,2 \pm 0,15 \mathrm{a}$ & 94,3 & $1,0 \pm 0,00 \mathrm{ab}$ & 16,7 \\
Enxofre & 500 & $1,8 \pm 0,48 \mathrm{a}$ & $5,5 \pm 0,64 \mathrm{a}$ & 1,5 & $1,5 \pm 0,29 \mathrm{a}$ & 52,4 & $1,0 \pm 0,41 \mathrm{ab}$ & 7,4 \\
Espirodiclofeno & 30 & $1,0 \pm 0,41 \mathrm{a}$ & $0,0 \pm 0,00 \mathrm{c}$ & 100,0 & $0,0 \pm 0,00 \mathrm{a}$ & 100,0 & $0,2 \pm 0,25 \mathrm{~b}$ & 66,7 \\
\hline
\end{tabular}

${ }^{1}$ Dosagem do produto comercial em g ou $\mathrm{mL}$ por 100 litros de água.

Médias ( \pm EP) seguidas por letras distintas, na coluna, diferem entre si, pelo teste de Tukey $(\mathrm{P}<0,05)$. Dados originais, para análise estatística, foram transformados em $\sqrt{\mathrm{x}+1,0}$.

${ }^{2}$ Percentual de eficiência dos agrotóxicos segundo a fórmula de Henderson e Tilton (1955).

\section{CONCLUSÕES}

1-No outono, Calepitrimerus vitis é controlado eficientemente por espirodiclofeno $\left(7,2\right.$ g i.a. $\left.100 \mathrm{~L}^{-1}\right)$ e por cihexatina $\left(30\right.$ g i.a. $\left.100 \mathrm{~L}^{-1}\right)$.

2-O acaricida-fungicida enxofre (400 g i.a. $100 \mathrm{~L}^{-1}$ ) controla eficientemente $C$. vitis na primavera, com uma única pulverização no outono ou com duas pulverizações, sendo uma no outono e outra na primavera.

3-Os acaricidas abamectina $\left(1,44\right.$ g i.a. $\left.100 \mathrm{~L}^{-1}\right)$ e espirodiclofeno $\left(6 \mathrm{~g}\right.$ i.a. $\left.100 \mathrm{~L}^{-1}\right)$ controlam eficientemente $C$. vitis na primavera com uma única pulverização na primavera ou com duas pulverizações, sendo uma no outono e outra na primavera.

4-Calepitrimerus vitis é controlado eficientemente com pulverizações de cihexatina, enxofre ou espirodiclofeno no outono ou durante a primavera com abamectina, enxofre ou espirodiclofeno.

\section{REFERÊNCIAS}

\section{AGROFIT. Sistema de agrotóxicos fitossa-} nitários. Brasília: Ministério da Agricultura, Pecuária e Abastecimento. Disponível em: $<$ http://extranet.agricultura.gov.agricultura. gov.br/agrofit_cons/principal_agrofit_cons $>$. Acesso em: 04 abr. 2010.

BERNARD, M.; HORNE, P.A.; HOFFMANN, A.A. Integrated management of grapevine rust mite (Calepitrimerus vitis) in Australian vineyards. In: EMMETT, B. (Org.). Strategic use of sulphur in integrated pest and disease management (IPM) programs for grapevines. Victoria: Department of Primary Industries/Primary Industries and Resources and Resources South Australia/Centre for Environ- mental Stress and Adaptation Research/La Trobe University, 2003b. p.182-188.

BERNARD, M.; HORNE, P.A.; HOFFMANN, A.A. Movement, distribution and effects of rust mite (Calepitrimerus vitis) and preliminary studies of bud mite (Colomerus vitis) on grapevines. In: EMMETT, B. (Org.). Strategic use of sulphur in integrated pest and disease management (IPM) programs for grapevines. Victoria: Department of Primary Industries/Primary Industries and Resources and Resources South Australia/Centre for Environmental Stress and Adaptation Research/La Trobe University, 2003a. p.133-152.

BRITO, H.M.; GONDIM JÚNIOR, M.G.C.; OLIVEIRA, J.V. de; CÂMARA, C.A.G. da. Toxicidade de Natuneem sobre Tetranychus urticae Koch (Acari: Tetranychidae) e ácaros predadores da família Phytoseiidae. Ciência e Agrotecnologia, Lavras, v.30, n.4, p.685-691, 2006.

CARMONA, M.M.; DIAS, J.C.S. Fundamentos de acarologia agrícola. Lisboa: Fundação Calouste Gulbenkian, 1996. 423p.

DANIEL, C.; LINDER, C.; WYSS, E. Autumn acaricide applications as a new strategy to control the pear leaf blister Eriophyes pyri. Crop Protection, Guildford, v.26, n.10, p.1532-1537, 2007.

DE LILLO, E.; MONFREDA, R.; BALDACHINNO, F. Efficacy of fungicides and acaricides against Calepitrimerus vitis (NALEPA). Phytophaga, Catania, v.14, p.599-603. 2004. 
DUFOR, R. Grapes: organic production. United States: ATTRA - National Sustainable Agriculture Information Service, 2006. 44p. Disponível em: $<$ http://attra.ncat.org/new pubs/attra-pub/PDF/grapes.pdf?id=Iowa $>$. Acesso em: 15 mar. 2010.

DUSO, C.; CASTAGNOLI, M.; SIMONI, S.; ANGELI, G. The impact the eriophyoids on crops: recent issues on Aculus schlechtendali, Calepitrimerus vitis and Aculops lycopersici. Experimental and Applied Acarology, Amsterdam, v.51, n.1-3, p.151-168, 2010.

FERLA, N.J.; BOTTON, M. Ocorrência do ácarovermelho Panonychus ulmi (Koch) (Tetranychidae) associado à cultura da videira no Rio Grande do Sul, Brasil. Ciência Rural, Santa Maria, v.38, n.6, p.1758-1761, 2008.

GODOY, M.S.; CARVALHO, G.A.; CARVALHO, B.F.; LASMAR, O. Seletividade fisiológica de inseticidas em duas espécies de crisopídeos. Pesquisa Agropecuária Brasileira, Brasília, v.45, n.11, p.1253-1258, 2010.

HENDERSON, E.A.; TILTON, E.W. Tests with acaricides against the brown wheat mite. Journal of Economic Entomology, Lanham, v.48, n.1, p.157165, 1955.

HERNÁNDEZ RIESCO, S.; CABALEIRO SOBRINO, C.; JACAS MIRET, J.; MARTÍN LÓPEZ, B. El empleo de aceites minerales, vegetales y de pescado en el Control Integrado de plagas y enfermedades del viñedo. Boletín de Sanidad Vegetal Plagas, Madrid, v.28, n.2, p.223-237, 2002.

HLUCHÝ, M.; POSPÍŠIL, Z. Damage and economic injury levels of eriophyid and tetranychid mites on grapes in Czechoslovakia. Experimental and Applied Acarology, Amsterdam, v.14, n.2, p.95-106, 1992.

MANZONI, C.G.; GRÜTZMACHER, A.D.; GIOLO, F.P.; HARTER, W. da R.; MÜLLER, C. Seletividade de agrotóxicos usados na produção integrada de maçã para adultos de Trichogramma pretiosum. Pesquisa Agropecuária Brasileira, Brasília, v.41, n.10, p.1461-1467, 2006.

MORAES, G.J.; FLECHTMANN, H.W. Manual de acarologia: acarologia básica e ácaros de plantas cultivadas no Brasil. Ribeirão Preto: Holos Editora, 2008. 308p.
RAUDONIS, L. Comparative toxicity of spirodiclofen and lambdacihalotrin to Tetranychus urticae, Tarsonemus pallidus and predatory mite Amblyseius andersoni in a strawberry site under field conditions. Agronomy Research, Estonian-Lithuanian, v.4, sp. iss., p.317-322, 2006. Disponível em: $<$ http://www. eau.ee/ agronomy/vol04Spec/p4S39.pdf $>$. Acesso em: 04 mar. 2010.

REIS, P.R.; NETO, M.P.; FRANCO, R.A. Controle de Brevipalpus phoenicis (Geijskes, 1939) e Oligonychus ilicis (McGregor, 1917) (Acari: Tenuipalpidae, Tetranychidae) em cafeeiro e o impacto sobre ácaros benéficos. II - Spirodiclofen e Azocyclotin. Ciência e Agrotecnologia, Lavras, v.29, n.3, p.528537, 2005.

RODRÍGUEZ, L.S.; GAETE, L.A.; REVETRIA, F.C.; ARRIAGADA, C.M. Nuevas especies de acaros fitófagos asociados a la vid vinífera en Chile. Santiago: Universidad de Chile, 2008. 11p. Disponível em: $<$ http://www.google.com.br/search?q=cale pitrimerus + vitis $\& h l=$ pt. $B R \& l r=\&$ start $=20 \& a s=N>$. Acesso em: 20 dez. 2008.

ROGERS, M.E.; STANSLY, P.A.; CHILDERS, C.C.; McCOY, C.W.; NIGG, H.N. Florida citrus pest management guide: rust mites, spider mites, and other phytophagous mites. Gainsville: University of Florida, 2010. 8p. (ENY, 603). Disponível em: $<$ http://edis.ifas.ufl.edu/pdffiles/CG/CG00200.pdf $>$ Acesso em: 25 mar. 2010.

RUIZ, M.G.; MORAES, G.J. de. Mortalidade do ácaro predador Neoseiulus californicus (Acari: Phytoseiidae) em testes de toxicidade residual de inseticidas e acaricidas usuais em pomáceas. Revista Brasileira de Fruticultura, Jaboticabal, v.30, n.4, p.919-924, 2008.

SORIA, S. de J.; DAL CONTE, A.F. Bioecologia e controle das pragas da videira. Bento Gonçalves: Embrapa Uva e Vinho, 2005. 20p. (Circular Técnica, $63)$.

VAN LEEUWEN, T.; WITTERS, J.; NAUEN, R.; DUSO, C.; TIRRY, L. The control of eriophyoid mites: state of art and future challenges. Experimental and Applied Acarology, Amsterdam, v.51, n.1-3, p.205-224, 2010. 
VAN POTTELBERGE, S.; KHAJEHALI, J.; VAN LEEUWEN, T.; TIRRY, L. Effects os spirodiclofen on reproduction in a susceptible and resistant strain of Tetranychus urticae (Acari: Tetranychidae). Experimental and Applied Acarology, Amsterdam, v.47, p.301-309, 2009.

VIEIRA, M.R.; GOMES, E.C.; FIGUEIRA, J.C. Controle químico de Calacarus heveae Feres (Acari: Eriophyidae) em seringueira. BioAssay, Piracicaba, v.1, n.9, p.1-7, 2006.
WACHENDORFF, U.; NAUEN, R.; SCHNORBACH, H.J.; RAUCH, N.; ELBERT, A. The biological profile of spirodiclofen (Envidor $\left.{ }^{\circledR}\right)$ : a new selective tetronic acid acaricide. Pflanzenschutz Nachrichten Bayer, Leverkusen, v.55, n.73, p.149176, 2002.

WALTON, V.M.; DREVES, A.J.; GENT, D.H.; JAMES, D.G.; MARTIN, R.R.; CHAMBERS, U.; SKINKIS, P.A. Relationship between rust mites $\mathrm{Ca}$ lepitrimerus vitis (NALEPA), bud mites Colomerus vitis (Pagenstecher) (Acari: Eriophyidae) and short shoot syndrome in Oregon vineyards. International Journal Acarology, Abingdon, v.33, n.4, p.307-318, 2007. 